This item was submitted to Loughborough's Research Repository by the author.

Items in Figshare are protected by copyright, with all rights reserved, unless otherwise indicated.

\title{
Procedures used in electrokinetic investigations of surfactant-laden interfaces, liquid films and foam system
}

PLEASE CITE THE PUBLISHED VERSION

https://doi.org/10.1016/j.cocis.2018.09.001

\section{PUBLISHER}

(C) Elsevier

\section{VERSION}

AM (Accepted Manuscript)

\section{PUBLISHER STATEMENT}

This paper was accepted for publication in the journal Current Opinion in Colloid and Interface Science and the definitive published version is available at https://doi.org/10.1016/j.cocis.2018.09.001

\section{LICENCE}

CC BY-NC-ND 4.0

\section{REPOSITORY RECORD}

Sheik, Abdulkadir Hussein, Faraz Montazersadgh, Victor Starov, Anna Trybala, and Hemaka Bandulasena. 2018. "Procedures Used in Electrokinetic Investigations of Surfactant-laden Interfaces, Liquid Films and Foam System". figshare. https://hdl.handle.net/2134/35327. 


\section{Accepted Manuscript}

Procedures used in Electrokinetic investigations of surfactantladen interfaces, liquid films and foam system

Abdulkadir Hussein Sheik, Faraz Montazersadgh, Victor Starov, Anna Trybala, H.C. Hemaka Bandulasena

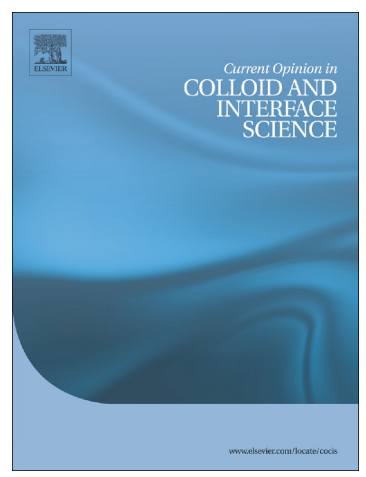

PII: $\quad$ S1359-0294(18)30007-4

DOI: $\quad$ doi:10.1016/j.cocis.2018.09.001

Reference: $\quad$ COCIS 1214

To appear in: $\quad$ Current Opinion in Colloid \& Interface Science

Received date: $\quad 31$ March 2018

Revised date: $\quad 24$ August 2018

Accepted date: $\quad 4$ September 2018

Please cite this article as: Abdulkadir Hussein Sheik, Faraz Montazersadgh, Victor Starov, Anna Trybala, H.C. Hemaka Bandulasena , Procedures used in Electrokinetic investigations of surfactant-laden interfaces, liquid films and foam system. Cocis (2018), doi:10.1016/j.cocis. 2018.09 .001

This is a PDF file of an unedited manuscript that has been accepted for publication. As a service to our customers we are providing this early version of the manuscript. The manuscript will undergo copyediting, typesetting, and review of the resulting proof before it is published in its final form. Please note that during the production process errors may be discovered which could affect the content, and all legal disclaimers that apply to the journal pertain. 


\title{
Procedures Used in Electrokinetic Investigations of Surfactant-Laden Interfaces, Liquid Films and Foam System
}

\author{
Abdulkadir Hussein Sheik ${ }^{1}$, Faraz Montazersadgh ${ }^{2}$, Victor Starov ${ }^{1}$, Anna Trybala ${ }^{1}$, \\ and H. C. Hemaka Bandulasena ${ }^{1 *}$ \\ ${ }^{1}$ Department of Chemical Engineering, Loughborough University, \\ ${ }^{2}$ Wolfson School of Mechanical, Manufacturing and Electrical Engineering, \\ Loughborough LE11 3TU, UK; A.Hussein-Sheik@lboro.ac.uk (A.H.S.); \\ F.Montazersadgh@lboro.ac.uk (F.M); V.M.Starov@lboro.ac.uk (V.S.) \\ A.Trybala@lboro.ac.uk (A.T.) *Correspondence: H.C.H.Bandulasena@lboro.ac.uk; \\ Tel.:+44-(0)-1509-222515
}

\section{Abstract}

Electrokinetic phenomena in liquid foam media are at a junction between two welldeveloped fields. On the one side is the study of liquid foam drainage, which is well documented, and on the other side is electrokinetics of surface driven flow on solidliquid interfaces, which is equally well understood. However, electrokinetic phenomena in foams with deformable air-liquid interfaces have gained significant attention only recently. In pursuit of understanding electrokinetics of foams, the model systems adopted by investigators can be summarised as: (i) free liquid films; (ii) flow cells (iii) a single bubble sandwiched between two electrodes; (iv) foam column and (v) numerical simulations. A new experimental approach for system monitoring and visualisation is proposed for foam electrokinetics. The results obtained from preliminary experiments are compared with the numerical simulations performed using Finite Element Method. The model predictions closely agree with the experimental data validating the model.

Keyword: Free liquid film; Liquid foam; Electroosmotic flow; Electrophoresis;

Electrokinetic;

\section{Introduction}

Liquid foams have a widespread application in cosmetics, detergents, food, firefighting, oil recovery and mining [1,2]. Foams are dispersed systems that consist of gas bubbles, which are partitioned by liquid films, Plateau borders and nodes. Foams are always formed in the presence of foaming agents (surfactants) as they stabilise liquid films between the bubbles. The equilibrium and the rate of adsorption are important characteristics of surfactants which determine stability of foams [3]. Drainage is a natural process driven by gravity and/or capillary action that occurs 
when the liquid between the gas bubbles flows mostly through Plateau borders (regions where three bubbles meet and make a connection of three liquid films at $120^{\circ}$ angle), and nodes (a connection between Plateau borders). The drainage processes are well investigated and documented. However, there is a new area of interest in studying the transfer processes in foams: the motion of liquid inside foams under the influence of an external electric field. Electrokinetic fluid transport through a foam system by an applied external electric field (electrokinetic flow) is complex and has not yet been fully understood [2]. Electrokinetic phenomena in foams are challenging due to the complex nature of hydrodynamic boundary condition and deformability of foam interfaces, which is generated at multiple scales [2]. Further challenges arise from electrokinetic transport at the charged liquid-vapour interface located on the bubble surfaces and solid-liquid interfaces. Charge is carried by ionic surfactants, and contribute substantially to surface elasticity [2,4]. The adsorption of ionic surfactant molecules at the respective interfaces governs the values of the zeta potential [2]. Publications on manipulation of fluids inside foam systems and wet films have increased considerably over the recent years. Below, various methods utilised by investigators for understanding foam electrokinetics are reviewed. Researchers have devised various experimental procedures to understand foam electrokinetics. These experimental approaches can be subdivided as follows: (i) free liquid films [5]; (ii) flow cells [4]; (iii) a single bubble [6]; (iv) foam column [7] and (v) numerical simulations [8].

\section{Surfactants used in electrokinetics flow experiments}

Surfactants reduce the interfacial tension by adsorption at the interface. Surfactants (or surface-active agents) are amphiphilic organic compounds. A surfactant molecule possesses a hydrophobic tail that is usually a long chain of hydrocarbons and a hydrophilic head that is a polar group (ionic or non-ionic). The amphiphilic nature of the surfactants gives rise to characteristic properties which are grouped as selfassembly and adsorption [3]. The hydrophilic head favours inclusion in water phase while hydrophobic tail favours inclusion in gas phase. Self-assembly is a feature of surfactants in an aqueous solution to arrange themselves into an organised structure referred to as micelles and/or vesicles, once the critical concentration is reached/exceeded. Often, this concentration is referred to as critical micelles concentration (CMC). At concentrations below $\mathrm{CMC}$, surfactant molecules form the solutions get adsorbed at the interfaces. At concentrations above CMC, the whole interface is completely covered with surfactants, and micelles start to form [3], as shown in Figure 1.

Cationic surfactants possess positively charged head groups: frequently a nitrogen atom or an amide group, and anionic surfactants possess a negatively charged head group: frequently a sodium ion [9]. The two groups of surfactants (cationic and anionic) are frequently used to create a surface charge. However, the use of nonionic surfactants can produce an advantage over the ionic ones, which includes dampening the effect of $\mathrm{pH}$ and controlling the solubility through manipulation of 
polarity and size of the head group. Zwitterionic (or amphoteric) surfactants can form both positively and negatively charged functional head group under specific conditions. Different type of surfactants that have been used in recent electrokinetic investigations on deformable surfaces are given in Table 1. A mixture of sodium lauryl sulfate (SDS) and cosurfactant dodecanol has been reported to improve foam stability [10].

Table 1: Summary of surfactants and experimental conditions used in electrokinetic flow experiments involving foam and deformable liquid films.

\begin{tabular}{|c|c|c|c|}
\hline Surfactant Type & Concertation(mM) & $\begin{array}{c}\text { Electric field } \\
(\mathrm{V} / \mathrm{m})\end{array}$ & Power type \\
\hline \multirow{4}{*}{$\begin{array}{l}\text { Sodium lauryl sulfate (SDS) } \\
\text { (anionic) }\end{array}$} & 34.7 [11] & $0-300 k$ & $\begin{array}{c}\text { AC (up to } 80 \\
\text { kHz) }\end{array}$ \\
\hline & $4-16[7]$ & 0 and 1000 & $D C$ \\
\hline & $8.2[8,12]$ & $254-4450$ & $\mathrm{DC}$ \\
\hline & $8.2[2]$ & $1000-12000$ & $\mathrm{DC}$ \\
\hline \multirow{4}{*}{$\begin{array}{c}\text { (Trimethyl(tetradecyl) } \\
\text { ammonium bromide (TTAB) } \\
\text { (cationic) }\end{array}$} & $8.9[6]$ & $0-5464$ & DC \\
\hline & $0.9[10]$ & - & $\mathrm{AC} / \mathrm{DC}$ \\
\hline & $5[8,12]$ & $254-4450$ & $\mathrm{DC}$ \\
\hline & $\begin{array}{c}2.25 \times 10^{-3}- \\
13.5[4]\end{array}$ & $0-3000$ & $\begin{array}{c}\text { AC (up to } 100 \\
\mathrm{~Hz}\end{array}$ \\
\hline $\begin{array}{l}\text { Dodecyltrimethylammonium } \\
\text { Bromide (DTAB) (cationic) }\end{array}$ & $5-30[7]$ & 0 and 1000 & DC \\
\hline $\begin{array}{c}\text { tetraethylene glycol } \\
\text { monooctyl ether (C8E4) } \\
\text { (non-ionic) }\end{array}$ & & 0 and 1000 & DC \\
\hline Commercial soap & Small amount [13] & $10-100 k$ & $\begin{array}{l}\text { AC (up to } 40 \\
\text { kHz) }\end{array}$ \\
\hline
\end{tabular}

The experimental set-ups and surfactants presented in Table 1 uses an electric field to manipulate the fluid flow. As a consequence, the liquid temperature increases due to Joule heating as current passes through the electrolyte solution [14]. This could result in a temperature gradient, both in the cross-stream and axial directions, causing non-uniform fluid properties. This could also affect mass transport of species through the foam/film. To our knowledge, the impact of Joule heating on liquid foams and liquid film electrokinetics has not been fully investigated yet.

\section{Electrokinetic experimental techniques}

A convenient model system for investigating electrokinetic phenomena in the presence of surfactant-laden interfaces is a free liquid film, which is formed by suspending a small volume of liquid stabilised by surfactants within a solid frame. In the case of a horizontal frame, the free liquid film is held by a frame from the sides while the top and bottom boundaries of the film are exposed to the atmosphere as shown in Figure 1(a). The supporting frame can be a square [13], rectangular [5] or 
circular [15]. The non-electrode part of the frame can be made from a range of materials such as borosilicate glass [8,12], printed circuit board [16] or aluminium rods covered with a dielectric material, which enables parts of the frame to act as electrodes when an electric field is applied [7]. A free liquid film can be used to model electrokinetic phenomenal in liquid foam systems as it captures unique geometric features of foam depending on the film thickness: foam films, Plateau borders or nodes. Figure 1(a) shows adsorption of an anionic surfactant to a gasliquid interface and to a positively charged substrate due to electrostatic attraction [17].

Liquid drainage of a vertical free liquid film counterbalanced by electrokinetic flow was reported by [7]. The free liquid film frame had dimensions of $4 \mathrm{~cm} \times 4 \mathrm{~cm} \times$ $0.087 \mathrm{~cm}$, and a range of voltages $(0 \mathrm{~V}-1000 \mathrm{~V})$ were applied using a DC power supply. The solutions were made by mixing water with either cationic, anionic or nonionic surfactants with a wide range of surfactant concentrations, either above or below CMC. To make the liquid film, the frame was dipped in the surfactant solution and withdrawn carefully. In this study [7], the importance of the charge of the surfactant molecules with regards to its interaction with the electric field and the electrical double layer (EDL) formation was demonstrated. The adsorption of ionic surfactant molecules at the interface governs the physics of the system, and by changing the direction of the electric field, the authors observed the subsequent effects on fluid flow and drainage. The local film thickness depends on the surfactant type used due to their interaction with the electric field. An important observation from this study [7] was that by changing the surfactant type from anionic to cationic, electroosmotic flow direction was completely reversed. The film was stabilised by two competing effects: the traction imposed by the electroosmotic flow and the pressure build-up at the end of the frame. The non-ionic surfactant showed marginal effects on the fluid flow field and film stability. Additionally, this study demonstrated the applicability of electrical stabilisation to foam in a gravity settler.

A liquid film motor is an experimental set-up where an external electric field (AC or DC) is applied to a free liquid film [13,15,18,19], and electrically induced rotation of the liquid is achieved by Electro-Hydrodynamical (EHD) effect. The free liquid film frame is kept horizontal in most cases to reduce the effect of the gravity. The experimental setup of a liquid film motor mainly consists of a freely suspended film placed between two capacitively coupled plates. In most cases, solutions were prepared by using water, glycerol and detergents. Typically, free liquid films are formed by submerging the supporting fame in the surfactant rich solution and withdrawing it carefully. Then the frame is placed in between two capacitor plates connected to a high voltage supply. Different frame geometries such as square [20], rectangular [13] or circular [16] have been tested to understand the impact of the geometry. 
(a)

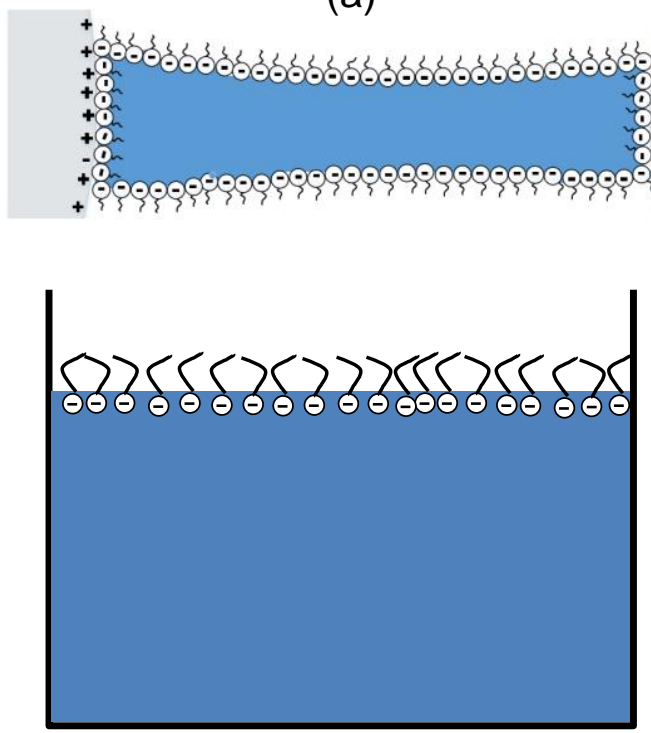

(c)

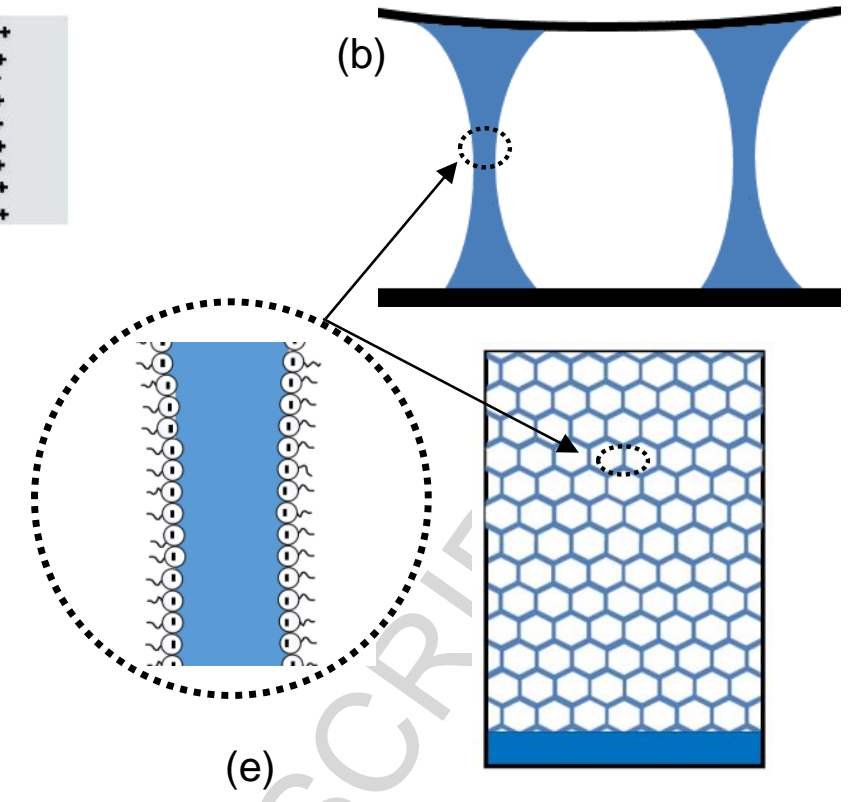

(d)

Figure 1: Illustration of configuration of different experimental setups used in foam electrokinetics. (a) a free liquid film $[5,7,8,12,13]$ (b) a single foam bubble sandwiched between two electrodes creating a cylindrical bubble $[6,10]$ (c) experimental cell created by adding surfactants in a transparent dish forming an adsorbed surfactant-laden liquid-gas interface [4,21] (d) a foam cell [2,7] (e) adsorption of surfactant molecules at the interface.

Ref $[8,12]$ reported measurement of electroosmotic flow in a free liquid film with a higher liquid content compared to that of ref [7]. The free liquid film thickness at its thinnest region was $180 \mu \mathrm{m}$, and the orientation of the film was kept horizontal to minimise the impact of gravity. However, since the length of the film in y-direction was greater than the capillary length, the effect of the gravity could not be neglected completely. To reduce the liquid drainage and to stabilise the liquid films, the authors used cationic and anionic surfactants with aqueous glycerol mixture in their study. A free liquid film was formed within the gap between the rods by taking $\sim \mu \mathrm{L}$ of the solution using a micropipette (Eppendorf, Hamburg, Germany), placing it on the cavity of the frame and gently moving it across to the other end. From this setup, the authors demonstrated that the direction and the features of electroosmotic flow depend on the type of surfactant used. This experimental setup allowed study of the complex hydrodynamic flow due to the combined effect of electroosmotic flow and the back pressure build-up. Depthwise velocity profiles obtained on this setup using micron-resolution particle image velocimetry were compared with the numerical simulations to validate the model developed. In ref [11], solutions were made with SDS surfactants at a concentration of $34.7 \mathrm{CMC}$ and $10 \%$ glycerol in water. To create a free liquid film, a rod wetted with the solution was drawn over the frame. 
The film thickness varied between $10 \mu \mathrm{m}$ to $10 \mathrm{~nm}$. A non-uniform electric field was applied.

In $[6,10]$, an experimental setup was developed for studying the electrokinetic phenomena in a single foam bubble stabilised by cationic surfactants. The bubble was sandwiched between two flat-plate electrodes, which resulted in formation of a cylindrical shape bubble as shown in Figure 1(b). The bubble was placed inside a hermetic box with a saturated vapour to prevent liquid evaporation from this film. The electroosmotic flow evolution of the bubble was monitored by conductivity measurements. This method involved measuring the liquid content of the cylindrical bubble for different applied electric fields across the film. Film thinness was inferred from the conductivity measurements. However, local film thickness variations of the film were neglected as only a single value of conductivity was recorded. Nonetheless, the proposed setup $[6,10]$ could be used to model interaction between foam films and Plateau borders. Electroosmotic flow resulted in build-up of liquid at the top end of the cylindrical bubble. A high salt concertation (1 mM-1 M) [10] was used in these experiments to drive the electroosmotic flow.

The experimental setup reported recently in [4] is a flow cell generated by filling a surfactant solution into a transparent dish forming a surfactant-laden gas-liquid interface as shown in Figure 1(c). The dimensions of the experimental cell were selected to be $10 \mathrm{~mm} \times 10 \mathrm{~mm}$ with a minimum depth of $3.7 \mathrm{~mm}$ to avoid interaction of surfactants with the bottom of the dish. The region of interest was the top surface, where the gas-liquid interface was covered with surfactants.

The challenge of stabilising liquid foam under an external electric field has led to the need of devising various experimental configurations for single liquid films as discussed above. However, manipulation of liquid foam under external electric fields has also been reported [2,7]. In [7], a liquid foam was generated in a column (as shown in Figure 1(d)) using an anionic surfactant (SDS) and placed in between two aluminium electrode plates. The authors noted electroosmotic flow similar to that observed in a vertical free liquid film as discussed in [7]. In ref [2], reverse drainage was reported in a foam column subjected to an external electric field. Foam was generated by bubbling air in an SDS solution at CMC. The bubble radius was approximately $1.5 \mathrm{~mm}$, and the tank height was $20 \mathrm{~cm}$. To avoid electrochemical reactions, electrodes were covered with Agar/Agar gel. To apply the electric field, one end of the electrode was submerged inside the solution reservoir while the other end was placed inside the foam. The applied electric field strength ranged from 1000 - $12000 \mathrm{~V} / \mathrm{m}$. Specific conductance was monitored over time [2] to determine the instantaneous liquid fraction in foam. The data revealed three distinctive regions. A 
quick reduction in liquid content characterised the initial region due to foam drainage. However, the rate was reduced gradually with the aid of compensating electroosmotic flow in the opposite direction. The measured conductance value reached a constant value depending on the electric field applied. For a sufficiently high electric field, flow was reversed [2]. The foam collapsed after a certain time, which was characterised by a sudden drop in instantaneous conductance. However, the flow direction reported by [2] is different from that reported in ref [7], even though the type of surfactant used was the same.

Experimental results are vital in understanding electrokinetic phenomena in foams. Researchers have combined experimental studies with numerical simulations to understand underpinning physics. Furthermore, once validated using experimental results [4,12], simulations can be used to predict flow behaviour in foam systems. In ref [12], numerical simulations performed using COMSOL Multiphysics ${ }^{\mathrm{TM}}$ were validated using experimental results. COMSOL is a commercially available software based on Finite Element Method (FEM) that has been used for electrokinetic flow investigations in thick liquid films for modelling flow in Plateau borders. A numerical study of the electrical and hydrodynamic equations related to a free liquid film imposed with non-uniform AC electric field was also solved in ref [22] using COMSOL. In [23], electroosmotic flow in a liquid film covered with ionic surfactants was modelled using molecular dynamic (MD) simulations. The authors found that the zeta potential value can be greater or less than earlier predictions based on the surface coverage. A liquid film motor configuration was also simulated in ref [20] and validated using experimental results [13]. A good agreement was found between the numerical simulations and the experimental results [20]. In [18], simulations were undertaken to explain the theory of the liquid film motors and to extend the understanding.

\section{Monitoring and visualisation of electrokinetic phenomina}

Innovative monitoring procedures for investigation of electrokinetic phenomena in liquid foam and deformable liquid films have been reported recently. [7] used microinterferometric techniques in their experimental setup to monitor the variations in local film thickness within a free liquid film. The principle of this approach was to observe interference of light at film surfaces and to ascribe different colour patterns observed to the local film thickness. The main limitation of this approach is that the film needs to be thicker than $30 \mathrm{~nm}$ [24]. A similar method was used in [13] for liquid film motors and [7] for investigation of drainage of a vertical free liquid films. Others [10] have used conductivity measurements to infer film thickness throughout the foam and to track electrokinetic effects on their respective systems. It should be noted that there is difference between electrokinetic measurements and similarly designed conductometric characterization of foams and thin liquid films as described in $[25,26]$. 
Recently, [11] reported tracking the surface of a $10 \mu \mathrm{m}$ thick free liquid film by using particle flakes $(<50 \mu \mathrm{m})$ deposited on the film surface. In this study, particle image velocimetry (PIV) technique was used to observe formation of a jet by a non-uniform AC electric field. A recent article utilised two imaging technique [4]: (i) optical microflow velocimetry that is similar to PIV, where tracer particles mixed with the solution allowed monitoring of the movement of the liquid. This method allowed measurement of complex hydrodynamic flow below the gas-liquid interface; (ii) Second Harmonic Generation (SHG) to monitor surfactant-laden gas-liquid interface [21]. SHG is a nonlinear optical method of analysing surfaces by converting two photons of different fundamental frequencies into a single photon at the harmonic frequency [27]. This technique enables monitoring the mobility of surfactants adsorbed at the interface. From SHG, the authors found that the surfactants adsorbed at the gas-liquid interface is static in the presence of electrically driven flow [4]. This result is interesting and will be very useful in simplifying numerical models.

Using a free liquid film, [8] measured velocity field generated by an external DC electric field using confocal laser scanning microscopy (CLSM) micron-resolution particle image velocimetry ( $\mu$-PIV). The system consisted of CLSM scan head, argon-ion laser (wavelength $=488 \mathrm{~nm}, 100 \mathrm{~mJ})$, photomultiplier tubes $(256 \times 256$ pixels, 8-bit resolution) and a computer with Laser Sharp 2000 software as shown in Figure 2. For flow visualisation, the authors used $2 \mu \mathrm{m}$ diameter fluorescent seed particles, which were at least 100 times smaller than the minimum film thickness. The peak excitation wavelength of the particles was $\sim 488 \mathrm{~nm}$, and the emission peak wavelength was $\sim 530 \mathrm{~nm}$. Following the identification of the top and the bottom interfaces of the liquid film using rhodamine $B$ dye, flow field was optically scanned at different depths $30 \mu \mathrm{m}$ apart. Using Cytodex® 3 microcarrier beads (SigmaAldrich), the depth of focus was estimated to be $\sim 8.4 \mu \mathrm{m}$. Approximately ten consecutive image pairs with a field of view of $690 \mu \mathrm{m} \times 690 \mu \mathrm{m}$ were recorded at a frame rate of $2 \mathrm{~Hz}$ for each depth-wise plane. Acquired images were analysed using PIVlab 1.4 [28]. The analysis involved cross-correlation of the recorded images by selecting $32 \times 32$ pixel interrogation window with a 50 percent overlap, which led to a spatial resolution of $20 \mu \mathrm{m} \times 20 \mu \mathrm{m} \times 8.4 \mu \mathrm{m}$. This procedure allowed measurement of 2D electroosmotic velocity at different depths in a free liquid film. The analysed region was 3200 to $3884 \mu \mathrm{m}$ away from the cathode electrode. The PIV technique used in this study provided fine details of the flow field than that of the flow velocimetry technique used in ref [4].

\section{Current developments}

A modified setup $[8,12]$ and an improved numerical model to predict the electrokinetic flow behaviour in a free liquid film is presented below. In this case, dye electrophoresis in the presence of electroosmotic flow near the anode electrode was considered. Test solutions were prepared by mixing $50 \mathrm{~g}$ of Milli-Q deionised water 
with $50 \mathrm{~g}$ of glycerol (Sigma-Aldrich, UK), $89.3 \mathrm{mg}$ of trimethyl(tetradecyl)ammonium bromide (TTAB) and $200 \mu \mathrm{L}$ of $1 \mathrm{M}$ phosphate buffer solution (Sigma-Aldrich, UK). Furthermore, $5 \mathrm{mM}$ fluorescein isothiocyanate (FICT) and $5 \mathrm{mM}$ rhodamine $B$ was added to the test solution for flow visualization. A free liquid film was prepared as described in [12]. Then an electric potential difference of $12 \mathrm{~V}$ was applied across the film, and the dye movement was recorded for several minutes. The experimental set up is presented in Figure 2.

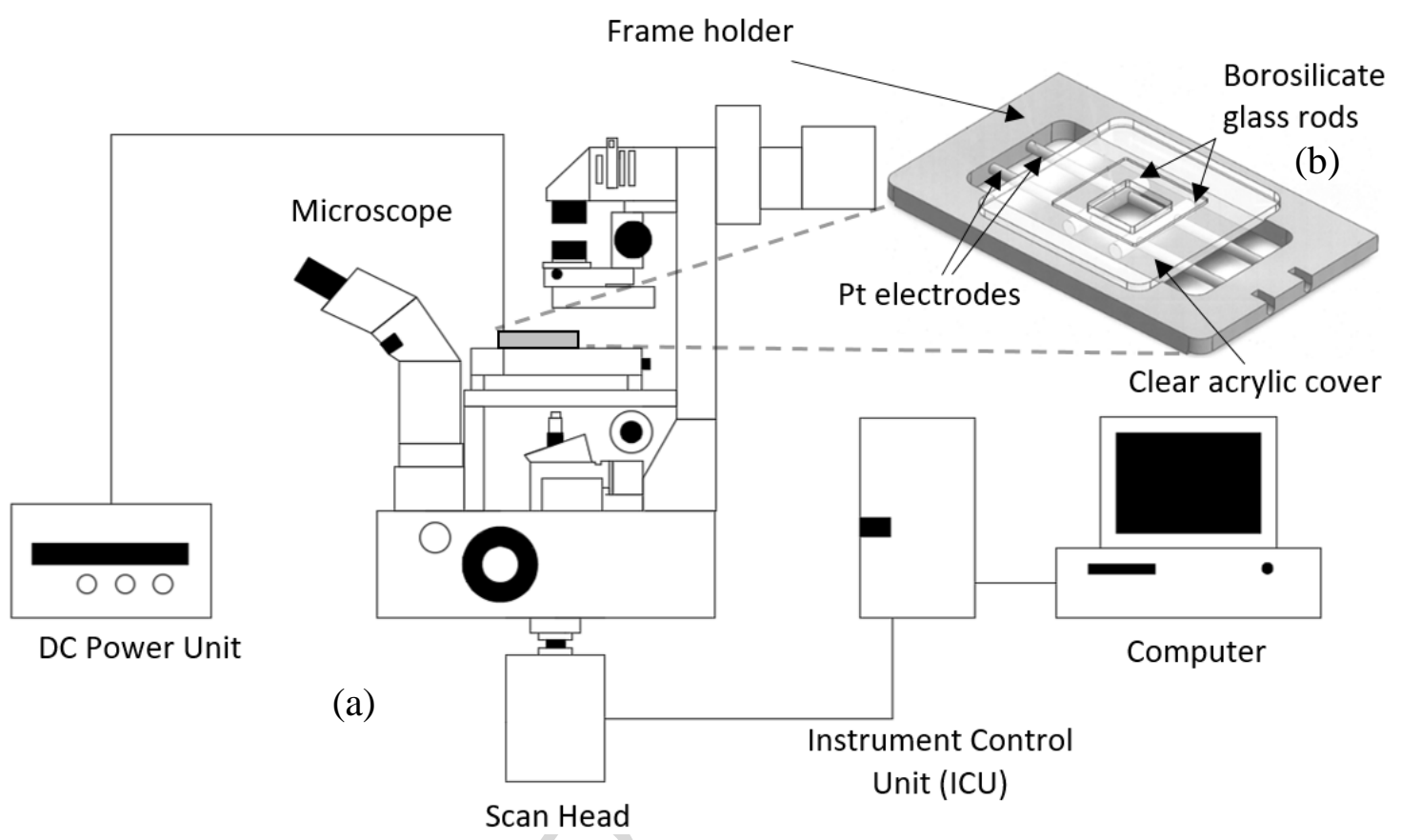

Figure 2: Experimental setup (a) confocal laser scanning microscopy (b) free liquid film frame holder.

Numerical simulations were performed using COMSOL 5.3a. The main equations solved are the continuity equation, Navier-Stokes equation, convection-diffusion for species transport and electric current conservation derived from Maxwell-Ampere's law. These equations were solved simultaneously for a 2D geometry with respective boundary conditions. The complex geometry was simplified for this purpose as shown in Figure 3.

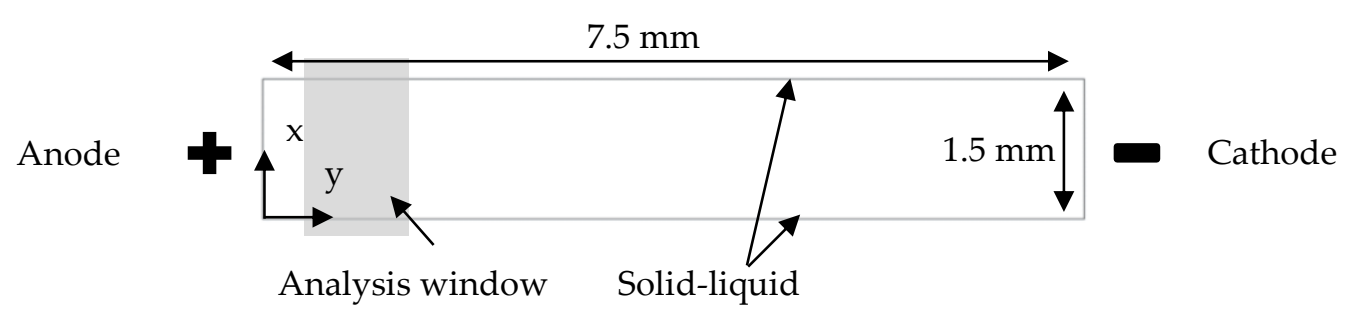


Figure 3: Computational domain. The analysis window is $\sim 200 \mu \mathrm{m}$ away from the anode electrode.

The chemical reactions that occurred within the flow domain included hydrolysis of water at the electrodes. The local $\mathrm{pH}$ within the domain was determined by the buffer reaction which can be described as:

$$
\mathrm{H}_{2} \mathrm{PO}_{4}{ }^{-} \rightleftharpoons \mathrm{H}^{+}+\mathrm{HPO}_{4}{ }^{2-}
$$

Fluorescein isothiocyanate (FICT) is extensively used for visualisation and separation of biological molecules in capillary electrophoresis and allied electrokinetics as its molecular structure enables labelling of biological molecules such as proteins, DNA or cells without damage. The dye undergoes protolytic reaction depending on the solution $\mathrm{pH}$ that gives rise to an overall charge of +1 (at highly acidic $\mathrm{pH} 2)$, neutral $(\mathrm{pH} \sim 4),-1$ and $-2(\mathrm{pH}>6.8)$ [29]. Under the experimental conditions, initial $\mathrm{pH}$ of the solution was fixed at 7.2 using a phosphate buffer concentration of $2 \mathrm{mM}$. At this $\mathrm{pH}$ value, the overall charge of the FICT is -2 . However, from the simulations, it was noted that the liquid film experienced local variation in $\mathrm{pH}$ throughout the domain. Near the anode, the $\mathrm{pH}$ was highly acidic. Therefore, the charge of the FICT molecules near the anode would be positive, which explains migration of the dye away from that electrode as shown in Figure 4(a). Furthermore, the electroosmotic flow towards the anode at the centre of the channel (the film is thin at the centre) and backflow due to pressure build up (where the film is thick) causes the dye front to form a complex shape. To compare this result with the numerical simulations, a range of charges were tested for FICT near the anode [29]. It was found that a charge of +0.1 for FICT provided a reasonable fit indicating a combination of different protolytic forms in that region due to local $\mathrm{pH}$ changes. The fitted charge agrees with that reported in the literature [29].

In predicting the slip velocity at the solid-liquid boundary, Helmholtz-Smoluchowski equation was used with a zeta potential value of $+70 \mathrm{mV}$ [12]. The estimated slip velocity was $1.1 \times 10^{-5} \mathrm{~m} . \mathrm{s}^{-1}$. However, a higher slip velocity of $2.0 \times 10^{-5} \mathrm{~m} . \mathrm{s}^{-1}$ provided a closer agreement with the experimental data. Even though a constant zeta potential was assumed for these simulations; it is possible that the zeta potential at the gas-liquid and solid-liquid interfaces are time and location dependent due to local $\mathrm{pH}$ changes. Furthermore, due to the simplification of the computational domain, three-dimensional effects of the flow field and the electric field were not captured in these computations. 
(a)

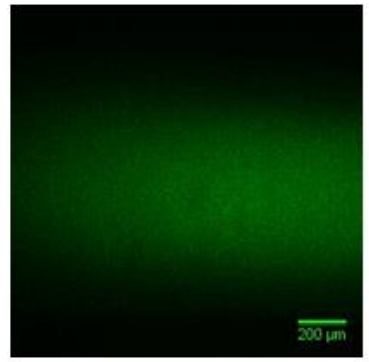

$0 \mathrm{~s}$

(b)

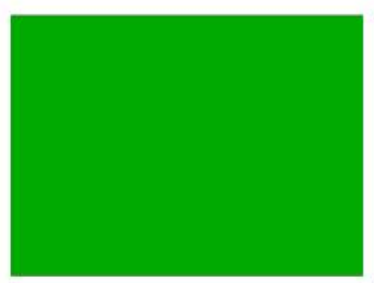

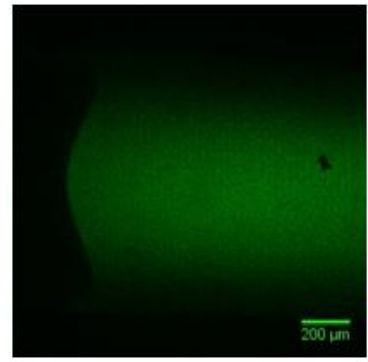

$14 \mathrm{~s}$

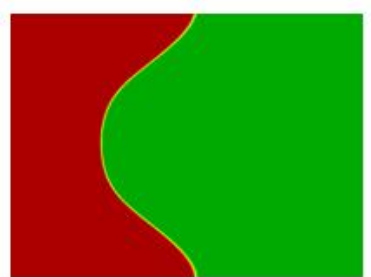

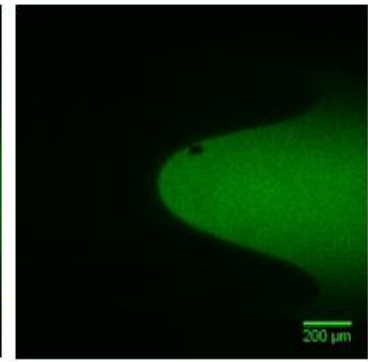

$28 \mathrm{~s}$

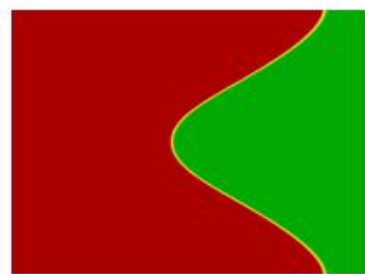

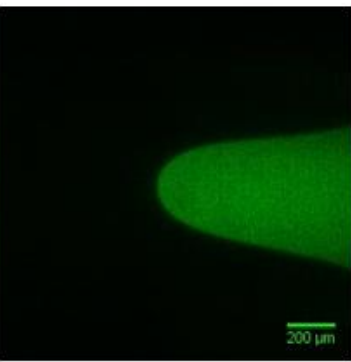

$33 \mathrm{~s}$

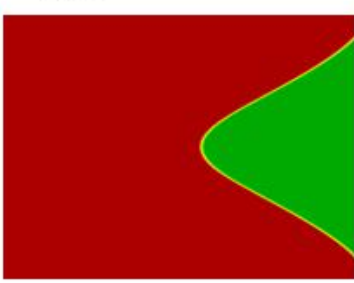

Figure 4: A comparison of experimental and numerical results for dye electrophoresis with the electroosmotic flow in a suspended film. Time evolution of FICT dye is shown by green colour (a) experimental results (b) numerical simulations. The analysis window is $\sim 200 \mu \mathrm{m}$ from the anode.

\section{Conclusions and outlook}

Foam electrokinetics has a potential use in many applications including separation of biological species such as proteins, DNA and RNA, and reverse drainage of foam. One of the key issues related to foam electrokinetic is foam instability under an external electric field, which leads to quick removal of fluid from foam films and Plateau borders. Therefore, surfactant formulation for foam stability under applied electric fields should be investigated further. In addition to that, computational models should be developed considering the complex 3D structure of foam and local variation of zeta potential with time.

Recent advances in the manipulation of fluid flow inside foam and deformable liquid surfaces using imposed electric field is summarised. These methods have been grouped as: (i) free liquid films; (ii) flow cells; (iii) a single bubble; (iv) foam column and (v) numerical simulations (mainly FEM). Since foam systems have a complex 3D geometry with varying local liquid content, some model systems and techniques used in the literature have advantages at studying different foam features (liquid foam films and Plateau borders). Moreover, a modified experimental approach for a free liquid film made from cationic or anionic surfactant solution mixture with charged dye was presented and discussed. The results were validated using a simplified 2D model solved using finite element method (FEM). A good agreement was found 
between the experimental results and the computer simulations. However, due to the complexity of the system, the data presented were limited to a single region of the film, and the simulated data represented a simplified 2D geometry of the free liquid film.

\section{Acknowledgements}

Authors would like to thank the Department of Chemical Engineering at Loughborough University for a doctoral scholarship for A.H.S.; Marie Curie CoWet project, EU; and MAP EVAPORATION, European Space Agency. The authors would also like to thank Tony Eyre for technical assistance.

\section{References}

[1] Arjmandi-Tash O, Trybala A, Mahdi FM, Kovalchuk NM, Starov V. Foams built up by non-Newtonian polymeric solutions: Free drainage. Colloids Surfaces $A$ Physicochem Eng Asp 2017;521:112-20.

[2] Bonhomme O, Blanc B, Joly L, Ybert C, Biance A-L. Electrokinetic transport in liquid foams. Adv Colloid Interface Sci 2017;247:477-90.

[3] M. R. Porter. Handbook of Surfactants. New York: Springer Verlag; 2013.

[4] Blanc B, Bonhomme O, Brevet P-F, Benichou E, Ybert C, Biance A-L. Electroosmosis near surfactant laden liquid-air interfaces. Soft Matter 2018;12:1-314.

[5] Hussein Sheik A, Bandulasena HCH, Starov V, Trybala A. Determining Electroosmotic Velocity in a Free Liquid Film. 20th Int Conf MTAS 2016:766-7.

[6] Bonhomme O, Liot O, Biance A-L, Bocquet L. Soft Nanofluidic Transport in a Soap Film. Phys Rev Lett 2013;110:054502.

[7] Sett S, Sahu RP, Sinha-Ray S, Yarin AL. Experimental Investigation of Eletrokinetic Stabilization of Gravitational Drainage of Ionic Surfactants Films. Electrochim Acta 2016;187:693-703.

[8] Hussein Sheik A, Bandulasena $\mathrm{HCH}$, Starov V, Trybala A. Electroosmotic flow measurements in a freely suspended liquid film: Experiments and numerical simulations. Electrophoresis 2017;0:1-7.

[9] Rosen MJ, Kunjappu J. Surfactants and Interfacial Phenomena. New Jersey: John Wiley \& Sons; 2012.

[10] Bonhomme O, Mounier A, Simon G, Biance A-L. Surface conductivity measurements in nanometric to micrometric foam films. J Phys Condens Matter 2015;27:194118.

[11] Shirsavar, R, Ramos, A, Amjadi, A, Taherinia, J, Mashhadir, M, Nejati A. Induced soap-film flow by non-uniform alternating electric field. J Electrostat 
2015;73:112-6.

[12] Hussein Sheik A, Trybala A, Starov V, Bandulasena $\mathrm{HCH}$. Electroosmotic Flow in Free Liquid Films: Understanding Flow in Foam Plateau Borders. Colloids and Interfaces 2018;2:8.

[13] Amjadi A, Shirsavar R, Hamedani R, Ejtehadi MR. A liquid film motor. Microfluid Nanofluid 2009;6:711-5.

[14] Abdollahzadeh Jamalabadi M. Joule heating in low-voltage electroosmotic with electrolyte containing nano-bubble mixtures through microchannel rectangular orifice. Chem Eng Res Des 2015;102:407-15.

[15] Amjadi A, Feiz MS, Namin RM. Liquid soap film generates electricity: a suspended liquid film rotating in an external electric field as an electric generator. Microfluid Nanofluidics 2015;18:141-7.

[16] Jiang S, Liu Z-Q, Tamar Amos Y, Kong X-M. Effects of Interfaces on Dynamics in Micro-Fluidic Devices : Slip-Boundaries ' Impact on Rotation Characteristics of Polar Liquid Film Motors. Commun Theor Phys 2017;67:577-89.

[17] Szymczyk K, Zdziennicka A, Ja B. Adsorption and wetting properties of cationic, anionic and nonionic surfactants in the glass-aqueous solution of surfactant-air system. Mater Chem Phys 2015;162:166-76.

[18] Feiz MS, Namin RM, Amjadi A. Theory of the liquid film motor. Phys Rev $\mathrm{E}$ 2015;92:1-9.

[19] Mollaei S, Nasiri M, Soltanmohammadi N, Shirsavar R, Ramos A, Amjadi A. Soap-film flow induced by electric fields in asymmetric frames. Phys Rev $E$ 2018;97:1-7.

[20] Nasiri M, Shirsavar R, Saghaei T, Ramos A. Simulation of liquid film motor: a charge induction mechanism. Microfluid Nanofluidics 2015;19:133-9.

[21] Blanc B, Bonhomme O, Joly L, Ybert C, Biance A. ELECTRO-OSMOSIS AT LIQUID INTERFACES. XXIV ICTAM, Montreal: 2016, p. 2-3.

[22] Nasiri M, Shirsavar R, Mollaei S, Ramos A. Numerical study of soap-film flow by nonuniform alternating electric fields. Phys Rev E 2017;95.

[23] Barbosa A, Lima D, Joly L. Electro-osmosis at surfactant-laden liquid-gas interfaces: beyond standard models. Soft Matter 2017;13:3341--3351.

[24] Sett S, Sinha-Ray S, Yarin AL. Gravitational Drainage of Foam Films. Langmuir 2013;29:4934-47.

[25] Karapantsios TD, Papara M. On the design of electrical conductance probes for foam drainage applications. Colloids Surfaces A Physicochem Eng Asp 2008;323:139-48.

[26] Kostoglou M, Georgiou E, Karapantsios TD. A new device for assessing film stability in foams: Experiment and theory. Colloids Surfaces A Physicochem Eng Asp 2011;382:64-73. 
[27] Matar G, Duboisset J, Benichou E, Bachelier G, Russier-Antoine I, Jonin C, et al. Second Harmonic Generation, a new approach for analyzing the interfacial properties of a short tryptophan-rich peptide. Chem Phys Lett 2010;500:161-6.

[28] Thielicke W, Stamhuis EJ. PIVlab - Towards User-friendly, Affordable and Accurate Digital Particle Image Velocimetry in MATLAB. J Open Res Softw $2014 ; 2$.

[29] Martin MM, Lindqvist L. THE pH DEPENDENCE OF FLUORESCEIN FLUORESCENCE. J Lumin 1975;10:381-90. 


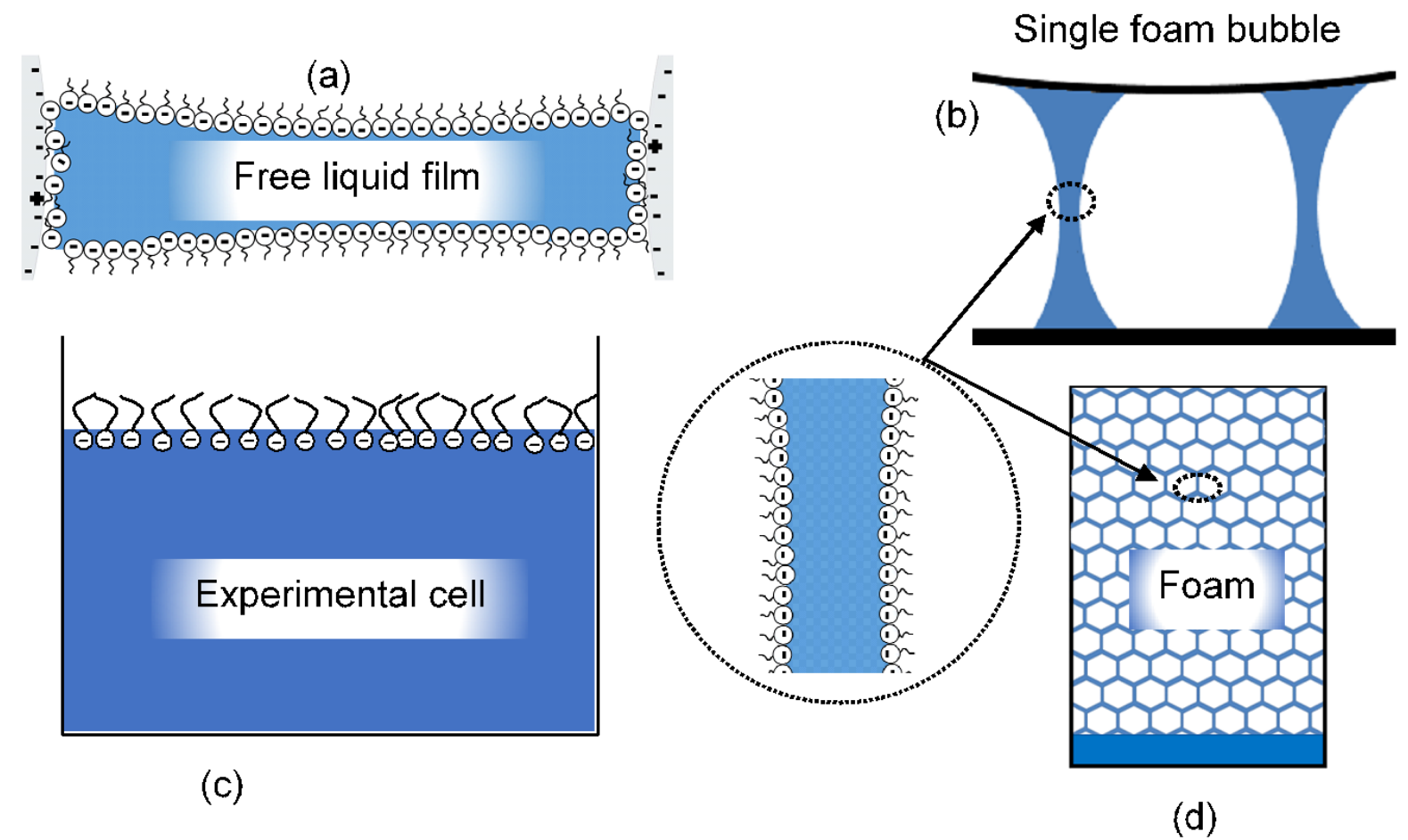

Graphical abstract 


\section{Highlights}

Electrokinetic phenomena in foams

Deformable interfaces

Slip velocity 